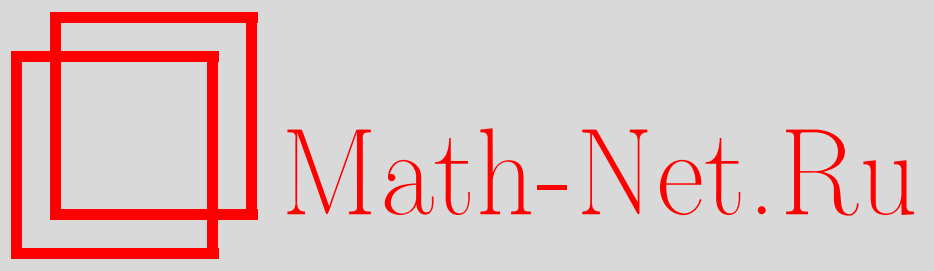

Ю. А. Изюмов, Н. И. Чащин, Исследование модели Хаббарда при половинном заполнении зоны, ТМФ, 2008, том 154, номер 1, 63-76

DOI: https://doi.org/10.4213/tmf6151

Использование Общероссийского математического портала Math-Net.Ru подразумевает, что вы прочитали и согласны с пользовательским соглашением http: //www . mathnet.ru/rus/agreement

Параметры загрузки:

IP: 54.92 .164 .108

26 апреля 2023 г., 15:42:15

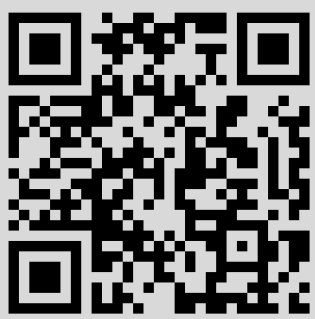




\title{
ИССЛЕДОВАНИЕ МОДЕЛИ ХАББАРДА ПРИ ПОЛОВИННОМ ЗАПОЛНЕНИИ ЗОНЫ
}

\begin{abstract}
В условиях сильного кулоновского отталкивания электронов на узле решетки исследованы уравнения для собственно-энергетической части электронной функции Грина, выведенные авторами ранее методом производящего функционала. Эти уравнения имеют форму, близкую к той, которая соответствует самосогласованному борновскому приближению в теории слабой связи. В полученных уравнениях далее опускается зависимость собственной энергии от импульса, что соответствует пределу бесконечной размерности пространства. Численное решение интегральных уравнений, где все величины зависят только от частоты, приводит к результатам, согласующимся с данными теории динамического среднего поля. Показано, в частности, что при увеличении кулоновского отталкивания трехпиковая структура квазичастичного спектра сменяется двухпиковой и имеет место фазовый переход металл-диэлектрик. Предложенный метод может быть применен при исследовании других моделей теории сильно коррелированных систем.
\end{abstract}

Ключевые слова: сильно коррелированные системы, модель Хаббарда, фазовый переход металл-изолятор.

\section{1. ВВЕДЕНИЕ}

Модель Хаббарда является одной из фундаментальных моделей в теории сильно коррелированных систем. Привлекательными являются ее простота и минимальная достаточность физического содержания. Действительно, гамильтониан содержит два слагаемых: один член описывает свободный перенос электрона по решетке, другой - кулоновское отталкивание электронов на одном узле. В представлении ферми-операторов рождения и уничтожения электронов гамильтониан имеет вид

$$
\mathcal{H}=\sum_{i j \sigma} t_{i j} c_{i \sigma}^{\dagger} c_{j \sigma}+U \sum_{i} n_{i \uparrow} n_{j \downarrow}
$$

Здесь $n_{i \sigma}=c_{i \sigma}^{\dagger} c_{i \sigma}$ - число электронов на узле $i$ со спином $\sigma=\uparrow, \downarrow$.

Модель содержит фактически два параметра: ширину затравочной зоны $W$ и кулоновский потенциал на узле $U$. В условиях сильной связи $(U \gtrsim W)$ два члена

* Институт физики металлов УрО РАН, Екатеринбург. E-mail: Yuri.Izyumov@imp.uran.ru 
гамильтониана отвечают за противоположные тенденции: к делокализации и локализации электронных состояний, и в зависимости от соотношения между $U$ и $W$ возможно образование либо металлического, либо диэлектрического состояния. Разумеется, в модели присутствует еще один параметр - концентрация электронов $n$, которая сильно влияет на реализацию фазового перехода в системе металл-изолятор. Известно, что наиболее благоприятные условия для такого фазового перехода возникают при половинном заполнении зоны $(n=1)$. В этом случае имеет место электронно-дырочная симметрия, и химический потенциал равен $\mu=U / 2$.

Модель Хаббарда в условиях сильной корреляции исследовалась многими способами. Особенно интересные результаты были получены в рамках теории динамического среднего поля (ТДСП), (см., например, обзор [1]), однако этот метод, игнорирующий пространственные корреляции и учитывающий только временны́е, имеет два недостатка. Сводя задачу о движении электрона на решетке к однопримесной проблеме, он требует значительных вычислительных ресурсов для решения последней. Другой недостаток проявляется, если в системе существенны пространственные корреляции; тогда необходимо разрабатывать расширенные схемы ТДСП. В силу отмеченных особенностей ТДСП определенную ценность имеют и другие подходы, например, использование уравнений движения для электронных функций Грина $(\Phi Г)$ в формализме $X$-операторов Хаббарда. Здесь можно отметить работы Плакиды с соавторами [2]-[4], в которых использовался метод Мори в статистической механике, и работы [5]-[7], опирающиеся на формализм Каданова и Бейма. Оба подхода используют самосогласованную теорию возмущений по электронному хоппингу и приводят к близким результатам, напоминающим схему самосогласованного борновского приближения (ССБП).

Недавно Плакида и Удовенко [4] провели численное решение уравнений ССБП для модели Хаббарда в представлении $X$-операторов для электронных концентраций за пределами половинного заполнения зоны с целью анализа электронных состояний в сверхпроводящих купратах. Следует отметить два интересных результата: был получен узкий квазичастичный пик в окрестности уровня Ферми между хаббардовскими подзонами и обнаружено частичное разрушение ферми-поверхности за счет образования псевдощелевого состояния. Эта работа показывает, что ССБП содержит эффекты пространственных корреляций, которые учитываются в ТДСП только при специальных расширениях метода.

Целью нашей работы является исследование модели Хаббарда при половинном заполнении зоны. Мы покажем, что в рамках приближения, близкого к ССБП, игнорируя пространственные корреляции, легко можно получить трехпиковую структуру спектра, которая была одним из первых достижений ТДСП; при этом достаточно использовать весьма скромные вычислительные ресурсы. При таком подходе становится весьма прозрачной физика системы, приводящая к эволюции от трехпиковой структуры спектра к двухпиковой при изменении кулоновского параметра $U$.

Вначале мы напомним, как вводится формализм $X$-операторов в модели Хаббарда. В условиях сильной корреляции гамильтониан (1.1) полезно выразить в терминах $X$-операторов (см., например, книгу [7]) и записать его в виде $\mathcal{H}=\mathcal{H}_{0}+\mathcal{H}_{1}$, 

где

$$
\begin{gathered}
\mathcal{H}_{0}=U \sum_{i} X_{i}^{22} \\
\mathcal{H}_{1}=\sum_{i j \sigma} t_{i j}\left(X_{i}^{\sigma 0}-\sigma X_{i}^{2 \bar{\sigma}}\right)\left(X_{i}^{0 \sigma}-\sigma X_{i}^{\bar{\sigma} 2}\right) .
\end{gathered}
$$

В этом представлении большой кулоновский член - одноузельный, что позволяет построить теорию возмущений вблизи атомного предела. Ферми-подобные операторы $X_{i}^{\sigma 0}$ и $X_{i}^{2 \bar{\sigma}}$ описывают рождение электрона на узле $i$, когда на нем не было электрона $\left(X_{i}^{\sigma 0}\right)$ и когда на узле был электрон с противоположным спином $\left(X_{i}^{2 \bar{\sigma}}\right)$, поэтому естественно ввести двухкомпонентные спиноры из этих операторов:

$$
\Psi(i \sigma)=\left(\begin{array}{c}
X_{i}^{0 \sigma} \\
\bar{\sigma} X_{i}^{2 \bar{\sigma}}
\end{array}\right), \quad \Psi^{\dagger}(i \sigma)=\left(X_{i}^{\sigma 0}, \bar{\sigma} X_{i}^{\bar{\sigma} 2}\right) .
$$

Тогда хоппинговский член гамильтониана записывается в компактном виде:

$$
\mathcal{H}_{1}=\sum_{i j \sigma} \sum_{\alpha \beta} \Psi_{\alpha}^{\dagger}(i \sigma) t_{\alpha \beta}(i j) \Psi_{\beta}(i \sigma) .
$$

Здесь $\Psi_{\alpha}^{\dagger}(i \sigma)$ представляет компоненту спинора $(\alpha=1,2)$, а

$$
t_{\alpha \beta}(i j)=t_{i j} \mathbb{T}_{\alpha \beta}, \quad \mathbb{T}=\left(\begin{array}{ll}
1 & 1 \\
1 & 1
\end{array}\right) .
$$

Мы видим, что в $X$-представлении теория имеет матричный характер по спинорным индексам, которые с самого начала отражают возможность возникновения двух хаббардовских подзон. Такой формализм широко используется в литературе.

В следующем разделе 2 вводится одночастичная электронная ФГ, построенная на $\Psi$-операторах, и приведены уравнения для неприводимой собственно-энергетической и концевой частей, полученные нами ранее в работах [5]-[7] в рамках теории возмущений вблизи атомного предела. Эти уравнения по своей структуре близки к уравнениям типа ССБП. Далее рассматривается случай половинного заполнения и налагается требование электронно-дырочной симметрии. Полученные итерациями уравнения упрощаются в духе приближения, возникающего в пределе бесконечной размерности пространства $(d \rightarrow \infty)$, а именно опускается зависимость собственной энергии от волнового вектора. Тогда получающаяся система нелинейных интегральных уравнений легко решается простыми численными методами. В разделе 3 вычисляется электронная плотность, которая показывает эволюцию от трехпиковой структуры к двухпиковой при увеличении $U$. Оцениваются вклады от поправок к членам ССБП-типа, а также проверяется правильность аналитических свойств электронной ФГ в условиях сильной корреляции.

\section{2. ЭЛЕКТРОННАЯ ФУНКЦИЯ ГРИНА В ТЕРМИНАХ $X$-ОПЕРАТОРОВ}

Информацию об одночастичных состояниях системы содержит электронная температурная $\Phi Г$

$$
g\left(1 \sigma_{1}, 2 \sigma_{2}\right)=-\left\langle T c_{1 \sigma_{1}} c_{2 \sigma_{2}}^{\dagger}\right\rangle .
$$

3 Теоретическая и математическая физика, т. 154, № 1, 2008 г. 
Здесь все обозначения стандартные: цифрами обозначены совокупные индексы, включающие узел и мацубаровское время, например, $1=\left(i_{1}, \tau_{1}\right) ; T$ - оператор временно́го упорядочения, а $\langle\ldots\rangle$ - символ статистического усреднения с гамильтонианом $\mathcal{H}$.

В условиях сильной корреляции для вычисления ФГ (2.1) удобно ввести вспомогательную матричную $Ф Г$, построенную на $X$-операторах:

$$
\mathcal{G}\left(1 \sigma_{1}, 2 \sigma_{2}\right)=-\left\langle T \Psi\left(1 \sigma_{1}\right) \Psi^{\dagger}\left(2 \sigma_{2}\right)\right\rangle
$$

В работах [5]-[7] разработана техника вычисления таких ФГ, основанная на уравнениях движения в терминах функциональных производных. Решение этих уравнений представляется в виде произведения двух величин:

$$
\mathcal{G}=G \Lambda
$$

где $G$ принято называть пропагаторной частью, а $\Lambda$ - концевой. Величина $G$ удовлетворяет уравнению Дайсона

$$
G^{-1}=G_{0}^{-1}-\Sigma
$$

где собственно-энергетическую часть можно представить в виде двух вкладов:

$$
\Sigma=\Sigma^{\prime}+\Lambda \mathcal{T}
$$

Для величин $\Sigma^{\prime}$ и $\Lambda$ выведены уравнения в функциональных производных, итерации в которых по хоппингу порождают теорию возмущений вблизи атомного предела, поскольку большое кулоновское взаимодействие полностью содержится в величине $G_{0}^{-1}$.

Приведем результаты вычисления $\Lambda$ и $\Sigma^{\prime}$ в пределах двух первых порядков по $t[6]$, [7], которые будут отправной точкой нашего исследования. Имеем для концевой части $\Lambda=\Lambda_{0}+\Lambda_{1}$, где

$$
\begin{gathered}
\Lambda_{0}^{\sigma}(k)=\left(\begin{array}{cc}
1-\left\langle n^{\bar{\sigma}}\right\rangle & 0 \\
0 & \left\langle n^{\bar{\sigma}}\right\rangle
\end{array}\right), \\
\Lambda_{1}^{\sigma}(k)=\left(\begin{array}{cc}
\lambda_{1}^{\sigma}(k) & \lambda_{2}^{\sigma}(k) \\
-\lambda_{1}^{\sigma}(k) & -\lambda_{2}^{\sigma}(k)
\end{array}\right), \\
\lambda_{1}^{\sigma}(k)=-\sum_{q} \varepsilon_{\mathbf{k}+\mathbf{q}}\left[\left(G_{11}^{\sigma}+G_{21}^{\sigma}\right)(k+q) \mathcal{N}^{\bar{\sigma} \bar{\sigma}}(q)+\right. \\
\left.+\left(G_{11}^{\bar{\sigma}}+G_{21}^{\bar{\sigma}}\right)(k+q) \mathcal{D}^{\sigma \bar{\sigma}}(q)+\left(G_{21}^{\bar{\sigma}}+G_{22}^{\bar{\sigma}}\right)(-k-q) \mathcal{D}^{02}(q)\right], \\
\lambda_{2}^{\sigma}(k)=\sum_{q} \varepsilon_{\mathbf{k}+\mathbf{q}}\left[\left(G_{12}^{\sigma}+G_{22}^{\sigma}\right)(k+q) \mathcal{N}^{\bar{\sigma} \bar{\sigma}}(q)+\right. \\
\left.+\left(G_{12}^{\bar{\sigma}}+G_{22}^{\bar{\sigma}}\right)(k+q) \mathcal{D}^{\sigma \bar{\sigma}}(q)+\left(G_{11}^{\bar{\sigma}}+G_{12}^{\bar{\sigma}}\right)(-k-q) \mathcal{D}^{02}(q)\right] .
\end{gathered}
$$


Величины $\mathcal{N}^{\bar{\sigma} \bar{\sigma}}(k), \mathcal{D}^{\sigma \bar{\sigma}}(k)$ и $\mathcal{D}^{02}(k)$ представляют собой фурье-компоненты бозонных $Ф Г$ :

$$
\begin{aligned}
\mathcal{N}^{\sigma_{1} \sigma_{2}}(12) & =-\left\langle T\left(n_{1}^{\sigma_{1}}-\left\langle n_{1}^{\sigma_{1}}\right\rangle\right)\left(n_{2}^{\sigma_{2}}-\left\langle n_{2}^{\sigma_{2}}\right\rangle\right)\right\rangle \\
\mathcal{D}^{\sigma \bar{\sigma}}(12) & =-\left\langle T X_{1}^{\sigma \bar{\sigma}} X_{2}^{\bar{\sigma} \sigma}\right\rangle \\
\mathcal{D}^{02}(12) & =-\left\langle T X_{1}^{02} X_{2}^{20}\right\rangle .
\end{aligned}
$$

В выражениях (2.6)-(2.10) $k$ и $q-4$-импульсы, например, $k=\left(\mathbf{k}, i \omega_{n}\right) ; \varepsilon_{\mathbf{k}}-$ фурье-компонента $t_{i_{1} i_{2}}$, представляющая энергию электрона в затравочной полосе; $n_{1}^{\sigma}=X_{1}^{\sigma \sigma}+X_{1}^{22}$.

Неприводимая собственно-энергетическая часть $\Sigma^{\prime}$ определяется выражением

$$
\Sigma^{\prime \sigma}(k)=-\left(\begin{array}{cc}
\eta_{1}^{\sigma}+\varphi_{1}^{\sigma}(k) & \eta_{2}^{\sigma}+\varphi_{2}^{\sigma}(k) \\
-\eta_{1}^{\sigma}-\varphi_{1}^{\sigma}(k) & -\eta_{2}^{\sigma}-\varphi_{2}^{\sigma}(k)
\end{array}\right)
$$

Здесь $\eta_{1}^{\sigma}$ и $\eta_{2}^{\sigma}$ являются величинами первого порядка по хоппингу $t$, не зависящими от 4-импульса ${ }^{1)}$ :

$$
\begin{aligned}
\eta_{1}^{\sigma} & =\sum_{k} \varepsilon_{\mathbf{k}}\left[G_{11}^{\sigma}(k)+G_{21}^{\sigma}(k)-G_{12}^{\sigma}(-k)-G_{22}^{\sigma}(-k)\right], \\
\eta_{2}^{\sigma} & =\sum_{k} \varepsilon_{\mathbf{k}}\left[G_{22}^{\sigma}(k)+G_{12}^{\sigma}(k)-G_{21}^{\sigma}(-k)-G_{11}^{\sigma}(-k)\right] .
\end{aligned}
$$

Величины $\varphi_{1}^{\sigma}(k)$ и $\varphi_{2}^{\sigma}(k)$ представляют собой поправку второго порядка по $t$ :

$$
\begin{aligned}
\varphi_{1}^{\sigma}(k) & =\sum_{q} \sum_{k_{1}} \varepsilon_{\mathbf{k}+\mathbf{q}} \varepsilon_{\mathbf{k}_{1}+\mathbf{q}}\left[G_{11}^{\sigma}\left(k_{1}\right) \bar{G}^{\bar{\sigma}}(k+q) G_{11}^{\bar{\sigma}}\left(k_{1}+q\right)+\right. \\
& \left.+G_{11}^{\bar{\sigma}}\left(k_{1}\right) \bar{G}^{\sigma}(k+q) G_{11}^{\bar{\sigma}}\left(k_{1}+q\right)-\sum_{\sigma^{\prime}} G_{22}^{\bar{\sigma}}\left(-k_{1}\right) \bar{G}^{\sigma^{\prime}}(k+q) G_{22}^{\bar{\sigma}^{\prime}}\left(-k_{1}-q\right)\right],
\end{aligned}
$$

a $\varphi_{2}^{\sigma}(k)$ получается заменой спинорных индексов в выражении $(2.13): 1 \leftrightarrow 2$. Здесь $\bar{G}^{\sigma}(k)$ - линейная комбинация матричных элементов пропагаторной $\Phi \Gamma$ :

$$
\bar{G}^{\sigma}(k)=G_{11}^{\sigma}(k)+G_{21}^{\sigma}(k)-G_{12}^{\sigma}(k)-G_{22}^{\sigma}(k) .
$$

Разрезаемая (по линии $t_{i j}$ ) часть $\Sigma$ представляет собой матрицу

$$
\Lambda^{\sigma} \mathcal{T}=\lambda^{\sigma}(k) \varepsilon_{\mathbf{k}}\left(\begin{array}{cc}
1 & 1 \\
-1 & -1
\end{array}\right)
$$

где

$$
\begin{aligned}
\lambda^{\sigma}(k) & =\lambda_{1}^{\sigma}(k)+\lambda_{2}^{\sigma}(k)= \\
& =-\sum_{q} \varepsilon_{\mathbf{k}+\mathbf{q}}\left[\bar{G}^{\sigma}(k+q) \mathcal{N}^{\bar{\sigma} \bar{\sigma}}(q)+\bar{G}^{\bar{\sigma}}(k+q) \mathcal{D}^{\sigma \bar{\sigma}}(q)-\bar{G}^{\bar{\sigma}}(-k-q) \mathcal{D}^{02}(q)\right] .
\end{aligned}
$$

1) В работах [6], [7] ошибочно сокращены недиагональные члены и приводится соотношение $\eta_{2}^{\sigma}=-\eta_{1}^{\sigma}=\sum_{k} \varepsilon_{\mathbf{k}}\left[G_{22}^{\sigma}(k)-G_{11}^{\sigma}(k)\right]$. 
Имея выражения (2.8)-(2.16), можно записать в явном виде пропагаторную ФГ:

$$
G^{\sigma}(k)=\left(G_{0}^{-1}(k)-\Sigma^{\prime \sigma}(k)-\Lambda^{\sigma} \mathcal{T}\right)^{-1},
$$

где

$$
G_{0}^{-1}(k)=\left(\begin{array}{cc}
i \omega_{n}+\mu & 0 \\
0 & i \omega_{n}+\mu-U
\end{array}\right) .
$$

Полную электронную $Ф Г \mathcal{G}$, определяемую соотношением $(2.2)$, получаем, согласно (2.3), перемножая матрицы $(2.17)$ и (2.6), (2.7). Электронную ФГ $g$, построенную на $c$-операторах, можно теперь вычислить с помощью соотношения

$$
g^{\sigma}(k)=\sum_{\alpha \beta} \mathcal{G}_{\alpha \beta}^{\sigma}(k) .
$$

Эти результаты согласуются с результатами Плакиды с соавторами [2]-[4], полученными в рамках приближения Мори. Действительно, вклад в разрезаемую собственно-энергетическую часть, даваемый выражениями (2.15), (2.16), совпадает по форме с представленным в этих работах и соответствует ССБП; различие заключается в члене $\Sigma^{\prime}$. Вклад первого порядка по $t$, определяющийся величинами $\eta_{1}^{\sigma}$ и $\eta_{2}^{\sigma}$, соответствует фоковскому члену приближения среднего поля (хартриевский член $\Lambda_{0} \mathcal{T}$ определяет приближение "Хаббард-I", если использовать терминологию теории возмущений по кулоновскому взаимодействию). В работах [2]-[4] также имеются поправки первого порядка к среднему полю, возникающие за счет статических флуктуаций заряда и спина; члены второго порядка по $t$, содержащие $\varphi_{1}^{\sigma}(k)$ и $\varphi_{2}^{\sigma}(k)$, отсутствуют. Если величины $\lambda^{\sigma}(k)$, соответствующие ССБП, учитывают взаимодействие фермиевских квазичастиц с бозонными (коллективными) степенями свободы, то член с $\varphi_{1}^{\sigma}(k)$ и $\varphi_{2}^{\sigma}(k)$ учитывает прямое взаимодействие фермионов друг с другом. Роль величин $\varphi_{1}^{\sigma}(k)$ и $\varphi_{2}^{\sigma}(k)$ будет выяснена позже.

\section{3. УРАВНЕНИЯ ДЛЯ ПАРАМАГНИТНОГО МЕТАЛЛА ПРИ ПОЛОВИННОМ ЗАПОЛНЕНИИ ЗОНЫ}

Рассмотрим далее парамагнитное состояние с половинным заполнением зоны $(n=$ $1)$, для которого $\mu=U / 2$. Разрешив матричное уравнение $(2.17)$, получим явное выражение для пропагаторной части электронной $Ф Г$ :

$$
G(k)=\frac{1}{d(k)}\left(\begin{array}{cc}
i \omega_{n}-\frac{U}{2}-\eta_{2}-\varphi_{2}-\left(\frac{1}{2}-\lambda\right) \varepsilon_{\mathbf{k}} & -\eta_{2}-\varphi_{2}+\left(\frac{1}{2}+\lambda\right) \varepsilon_{\mathbf{k}} \\
\eta_{1}+\varphi_{1}+\left(\frac{1}{2}-\lambda\right) \varepsilon_{\mathbf{k}} & i \omega_{n}+\frac{U}{2}+\eta_{1}+\varphi_{1}-\left(\frac{1}{2}+\lambda\right) \varepsilon_{\mathbf{k}}
\end{array}\right)
$$

где знаменатель определяется выражением

$$
d(k)=\left(i \omega_{n}\right)^{2}-\left(\frac{U}{2}\right)^{2}+\left(\eta^{-}+\varphi^{-}\right) i \omega_{n}-\frac{U}{2}\left(\eta^{+}+\varphi^{+}\right)-\left(i \omega_{n}-U \lambda+\eta^{-}+\varphi^{-}\right) \varepsilon_{\mathbf{k}} .
$$

Мы обозначили $\eta^{ \pm}=\eta_{1} \pm \eta_{2}, \varphi^{ \pm}=\varphi_{1} \pm \varphi_{2}$ и опустили аргумент $k$ у величин $\varphi^{ \pm}(k)$ и $\lambda(k)$; величины $\eta^{ \pm}$не зависят от $k$. 
Учитывая соотношение $(2.18)$, связывающее $\Phi \Gamma g$ с $\Phi \Gamma \mathcal{G}$, построенной на $X$-операторах, представим электронную ФГ в локаторной форме:

$$
g(k)=\frac{1}{F(k)-\varepsilon_{\mathbf{k}}},
$$

где локатор

$$
F(k)=\frac{\left(i \omega_{n}\right)^{2}-(U / 2)^{2}+\left(\eta^{-}+\varphi^{-}\right) i \omega_{n}-(U / 2)\left(\eta^{+}+\varphi^{+}\right)}{i \omega_{n}-U \lambda+\eta^{-}+\varphi^{-}} .
$$

С помощью спектрального представления для электронных и бозонных ФГ проведем суммирование по мацубаровским частотам в выражениях для $\eta, \lambda$ и $\varphi$ и сделаем аналитическое продолжение $i \omega_{n} \rightarrow \omega+i \delta$ к запаздывающим ФГ [8]. Тогда $\eta_{1}$ можно записать в виде

$$
\begin{aligned}
\eta_{1}=\frac{1}{N} \sum_{\mathbf{k}} \varepsilon_{\mathbf{k}} \frac{1}{\pi} \int_{-\infty}^{\infty} d \omega\left(\operatorname{Im}\left[G_{11}(\mathbf{k}, \omega)+G_{12}(\mathbf{k}, \omega)\right] f(\omega)+\right. \\
\left.\quad+\operatorname{Im}\left[G_{22}(\mathbf{k}, \omega)+G_{21}(\mathbf{k}, \omega)\right] f(-\omega)\right) .
\end{aligned}
$$

Формула для $\eta_{2}$ получается из (3.5) заменой спинорных индексов: $1 \leftrightarrow 2$.

Выпишем выражения для мнимых частей функций $\lambda(k)$ и $\varphi^{ \pm}(k)$ :

$$
\begin{aligned}
\operatorname{Im} \lambda(\mathbf{k}, \omega)=- & \frac{2}{\pi} \sum_{\mathbf{q}} \varepsilon_{\mathbf{k}+\mathbf{q}} \int_{-\infty}^{\infty} d \Omega \operatorname{Im} \bar{G}(\mathbf{k}+\mathbf{q}, \omega+\Omega) \operatorname{Im} \mathcal{D}(\mathbf{q}, \Omega) \times \\
& \times[f(\omega+\Omega)+N(\Omega)], \\
\operatorname{Im} \varphi^{ \pm}(\mathbf{k}, \omega)= & \frac{1}{\pi} \sum_{\mathbf{q}} \varepsilon_{\mathbf{k}+\mathbf{q}} \int_{-\infty}^{\infty} d \Omega \operatorname{Im} \bar{G}(\mathbf{k}+\mathbf{q}, \omega+\Omega) \operatorname{Im} m^{ \pm}(\mathbf{q}, \Omega) \times \\
& \times[f(\omega+\Omega)+N(\Omega)],
\end{aligned}
$$

где $f(\omega)$ и $N(\Omega)$ - функции Ферми и Бозе, соответственно. В последнем выражении введена величина, аналогичная мнимой части бозонной $\Phi \Gamma \mathcal{D}(\mathbf{q}, \Omega)$ :

$$
\begin{aligned}
\operatorname{Im} m^{ \pm}(\mathbf{q}, \Omega)=\frac{2}{\pi} & \sum_{\mathbf{k}^{\prime}} \varepsilon_{\mathbf{k}^{\prime}+\mathbf{q}} \int_{-\infty}^{\infty} d \omega^{\prime}\left[\operatorname{Im} G_{11}\left(\mathbf{k}^{\prime}+\mathbf{q}, \omega^{\prime}+\Omega\right) \operatorname{Im} G_{11}\left(\mathbf{k}^{\prime}, \omega^{\prime}\right) \pm\right. \\
& \left. \pm \operatorname{Im} G_{22}\left(\mathbf{k}^{\prime}+\mathbf{q}, \omega^{\prime}+\Omega\right) \operatorname{Im} G_{22}\left(\mathbf{k}^{\prime}, \omega^{\prime}\right)\right]\left[f\left(\omega^{\prime}+\Omega\right)-f\left(\omega^{\prime}\right)\right] .
\end{aligned}
$$

Реальные части величин $\lambda(\mathbf{k}, \omega)$ и $\varphi^{ \pm}(\mathbf{k}, \omega)$ могут быть найдены из мнимых частей c помощью дисперсионных соотношений.

Уравнения (3.6) и (3.7) являются интегральными по частотам и импульсам. Мы упростим их, используя основную идею ТДСП о том, что в пределе $d \rightarrow \infty$ собственно-энергетическая часть одночастичной $Ф Г$ не зависит от импульса. В данном приближении это означает, что величины $\lambda(\mathbf{k}, \omega)$ и $\varphi^{ \pm}(\mathbf{k}, \omega)$ не зависят от импульса $\mathbf{k}$, но зависят от частоты $\omega$. Уравнения для $\lambda(\omega)$ и $\varphi^{ \pm}(\omega)$ можно получить, если просуммировать выражения (3.6) и (3.7) по $\mathbf{k}$, а выражение (3.8) - по q. Таким образом, из (3.6) получаем

$$
\operatorname{Im} \lambda(\omega)=-\frac{2}{\pi} \int_{-\infty}^{\infty} d \Omega \operatorname{Im} \bar{G}^{[1]}(\omega+\Omega) \operatorname{Im} \mathcal{D}(\Omega)[f(\omega+\Omega)+N(\Omega)],
$$


где

$$
\begin{aligned}
\mathcal{D}(\Omega) & =\frac{1}{N} \sum_{\mathbf{q}} \mathcal{D}(\mathbf{q}, \Omega), \\
\bar{G}^{[1]}(\omega) & =\frac{1}{N} \sum_{\mathbf{k}} \varepsilon_{\mathbf{k}} \bar{G}(\mathbf{k}, \omega) .
\end{aligned}
$$

Уравнение аналогичного типа возникает, если просуммировать (3.7) по k:

$$
\operatorname{Im} \varphi^{ \pm}(\omega)=\frac{1}{\pi} \int_{-\infty}^{\infty} d \Omega \operatorname{Im} \bar{G}^{[1]}(\omega+\Omega) \operatorname{Im} m^{ \pm}(\Omega)[f(\omega+\Omega)+N(\Omega)],
$$

где

$$
\begin{aligned}
\operatorname{Im} m^{ \pm}(\Omega)=\frac{2}{\pi} & \int_{-\infty}^{\infty} d \omega\left[\operatorname{Im} G_{11}^{[1]}(\omega+\Omega) \operatorname{Im} G_{11}(\omega) \pm \operatorname{Im} G_{22}^{[1]}(\omega+\Omega) \operatorname{Im} G_{22}(\omega)\right] \times \\
& \times[f(\omega+\Omega)-f(\omega)]
\end{aligned}
$$

Здесь

$$
G_{\alpha \beta}(\omega)=\frac{1}{N} \sum_{\mathbf{k}} G_{\alpha \beta}(\mathbf{k}, \omega), \quad G_{\alpha \beta}^{[1]}(\omega)=\frac{1}{N} \sum_{\mathbf{k}} \varepsilon_{\mathbf{k}} G_{\alpha \beta}(\mathbf{k}, \omega) .
$$

Из формул (3.9) и (3.13) следуют условия симметрии для величин $\lambda(\omega)$ и $\varphi^{ \pm}(\omega)$ :

$$
\begin{aligned}
& \operatorname{Im} \lambda(-\omega)=\operatorname{Im} \lambda(\omega), \quad \operatorname{Im} \varphi^{ \pm}(-\omega)=\mp \operatorname{Im} \varphi^{ \pm}(\omega), \\
& \operatorname{Re} \lambda(-\omega)=-\operatorname{Re} \lambda(\omega), \quad \operatorname{Re} \varphi^{ \pm}(-\omega)= \pm \operatorname{Im} \varphi^{ \pm}(\omega) .
\end{aligned}
$$

Они выполняются, разумеется, только для половинного заполнения и являются следствием электронно-дырочной симметрии. Легко показать, что из требований электронно-дырочной симметрии $\eta_{1}=\eta_{2}=0$.

Таким образом, мы имеем систему интегральных уравнений для $\lambda(\omega)$ и $\varphi^{ \pm}(\omega)$, в которых предполагается, что $\operatorname{Im} \mathcal{D}(\Omega)$ задается отдельно. Эти уравнения нелинейные, поскольку пропагаторная $\Phi \Gamma G(k)$ содержит $\lambda(\omega)$ и $\varphi^{ \pm}(\omega)$ в знаменателе $d(k)$. Ниже будет показано, что величины $\varphi^{ \pm}(\omega)$ малы по сравнению с $U \lambda$, поэтому сейчас мы пренебрежем ими во всех уравнениях. В этом приближении уравнение (3.9) для $\lambda(\omega)$ можно записать в виде

$$
\operatorname{Im} \lambda(\omega)=U^{2} \int_{-\infty}^{\infty} d \Omega \operatorname{Im} \lambda(\omega+\Omega) Q(\omega+\Omega) \frac{1}{\pi} \operatorname{Im} \mathcal{D}(\Omega)[f(\omega+\Omega)+N(\Omega)],
$$

где

$$
\begin{gathered}
Q(\omega)=\frac{1}{N} \sum_{\mathbf{k}} \frac{\varepsilon_{\mathbf{k}}^{2}}{\operatorname{Re}^{2} d\left(\omega, \varepsilon_{\mathbf{k}}\right)+\operatorname{Im}^{2} d\left(\omega, \varepsilon_{\mathbf{k}}\right)}, \\
\operatorname{Re} d\left(\omega, \varepsilon_{\mathbf{k}}\right)=\omega^{2}-\left(\frac{U}{2}\right)^{2}-[\omega-U \operatorname{Re} \lambda(\omega)] \varepsilon_{\mathbf{k}}, \\
\operatorname{Im} d\left(\omega, \varepsilon_{\mathbf{k}}\right)=U \operatorname{Im} \lambda(\omega) \varepsilon_{\mathbf{k}} .
\end{gathered}
$$


При переходе от уравнения (3.9) к (3.16) мы учли, что $\bar{G}(k)=-U / d(k)$.

$\mathrm{K}$ уравнению (3.16) следует добавить дисперсионное соотношение, связывающее реальную и мнимую части величины $\lambda(\omega)$ :

$$
\operatorname{Re} \lambda(\omega)=-\frac{1}{\pi} \int_{-\infty}^{\infty} d \omega^{\prime} \frac{\operatorname{Im} \lambda\left(\omega^{\prime}\right)}{\omega-\omega^{\prime}} .
$$

Уравнения (3.16) и (3.19) представляют собой замкнутую систему уравнений для величин $\operatorname{Im} \lambda\left(\omega^{\prime}\right)$ и $\operatorname{Re} \lambda\left(\omega^{\prime}\right)$ при условии, что задана спектральная плотность спиновых флуктуаций $(1 / \pi) \mathrm{D}(\Omega)$.

Получим теперь выражение для плотности электронных состояний (на одну проекцию спина):

$$
\rho(\omega)=\frac{1}{N} \sum_{\mathbf{k}} \varepsilon_{\mathbf{k}}\left[-\frac{1}{\pi} \operatorname{Im} g\left(\varepsilon_{\mathbf{k}}, \omega\right)\right] .
$$

Согласно формуле (3.3)

$$
g\left(\varepsilon_{\mathbf{k}}, \omega\right)=\frac{1}{F_{1}(\omega)+i F_{2}(\omega)-\varepsilon_{\mathbf{k}}},
$$

где $F_{1}(\omega)$ и $F_{2}(\omega)$ - вещественная и мнимая части локатора $(3.4)$, который при условии $\eta_{1}=\eta_{2}=0$ и при пренебрежении величинами $\varphi^{ \pm}(\omega)$ оказывается равным

$$
F(\omega)=\frac{\omega^{2}-(U / 2)^{2}}{\omega-U \lambda(\omega)} .
$$

С другой стороны, одночастичная электронная ФГ (2.1) представима через собственно-энергетическую часть $\Sigma$ :

$$
g\left(\varepsilon_{\mathbf{k}}, \omega\right)=\frac{1}{\omega-\Sigma\left(\varepsilon_{\mathbf{k}}, \omega\right)+\mu} .
$$

Сравнивая соотношения (3.21) и (3.23), можно найти выражение для $\Sigma(\omega)$ через $\lambda(\omega)$; выпишем его для мнимой части $\Sigma(\omega)$ :

$$
\operatorname{Im} \Sigma(\omega)=-F_{2}(\omega)=-\frac{\left(\omega^{2}-(U / 2)^{2}\right) U \operatorname{Im} \lambda(\omega)}{[\omega-U \operatorname{Re} \lambda(\omega)]^{2}+[U \operatorname{Im} \lambda(\omega)]^{2}} .
$$

Для запаздывающей $\Phi \Gamma \operatorname{Im} \Sigma(\omega)<0$, поэтому для выполнения аналитических свойств электронной ФГ из выражения (3.24) следует необходимость неравенства

$$
\left(\omega^{2}-\left(\frac{U}{2}\right)^{2}\right) U \operatorname{Im} \lambda(\omega)>0,
$$

т.е. $\operatorname{Im} \lambda(\omega)$ должна менять знак в точках $\omega= \pm U / 2$, которые соответствуют положению атомных уровней при наличии кулоновского взаимодействия электронов. Условие (3.25) обеспечивает положительную определенность электронной плотности состояний. В случае прямоугольной затравочной полосы шириной $W$

$$
\rho_{0}(\omega)=\left\{\begin{array}{lll}
1, & \text { если } & |\omega|<W / 2, \\
0, & \text { если } & |\omega|>W / 2
\end{array}\right.
$$


интегрирование по $\varepsilon$ в формуле (3.20) приводит к выражению

$$
\rho(\omega)=\frac{1}{\pi}\left[\operatorname{arctg} \frac{1+2 F_{1}(\omega)}{2 F_{2}(\omega)}+\operatorname{arctg} \frac{1-2 F_{1}(\omega)}{2 F_{2}(\omega)}\right] .
$$

Нетрудно видеть, что $\rho(\omega)$ - четная функция $\omega$, т.е. при половинном заполнении имеет место электронно-дырочная симметрия.

\section{4. СТРУКТУРА ЭЛЕКТРОННОГО СПЕКТРА}

Из вида уравнения (3.16) следует, что оно имеет тривиальное решение $\lambda(\omega)=0$ и нетривиальное, причем если $\lambda(\omega)$ есть некоторое решение, то и $-\lambda(\omega)$ тоже будет решением этого уравнения. Можно также увидеть, что допускается решение, меняющее знак в точках $\omega= \pm U / 2$. Именно такие решения нам необходимы для выполнения условия аналитичности запаздывающей ФГ в верхней полуплоскости, требующего удовлетворения неравенства (3.25).

Для явного решения уравнения (3.16) необходимо задать спектральную функцию спиновых флуктуаций $\operatorname{Im} \mathcal{D}(\omega)$. Мы воспользуемся модельной аппроксимацией, предложенной в работе [9] и успешно примененной к аналогичной проблеме в работе [7]:

$$
\frac{1}{\pi} \operatorname{Im} \mathcal{D}(\Omega)=\chi_{0} \frac{\operatorname{th}(\Omega /(2 T))}{1+(\Omega / \Gamma)^{2}}
$$

Таким образом, введены два параметра $\chi_{0}$ и $\Gamma$, характеризующие интенсивность и ширину спектрального распределения, соответственно. Между ними существует связь, задаваемая тождеством

$$
\left\langle\mathbf{S}_{i}^{2}\right\rangle=\int_{-\infty}^{\infty} d \Omega \frac{1}{\pi} \operatorname{Im} \mathcal{D}(\Omega) N(\Omega)
$$

которая сводится к соотношению

$$
\pi \chi_{0} \Gamma=\frac{3}{2}
$$

Если подставить выражение (4.1), в котором $\chi_{0}=3 /(2 \pi \Gamma)$, в основное уравнение (3.16), то следует искать решения при заданных параметрах $U$ и $Г$. Уравнение (3.16) должно решаться совместно с уравнением (3.19). Решение этой системы интегральных уравнений находится итерациями, причем затравочное значение $\lambda(\omega)$ выбирается таким, чтобы в точках $\omega= \pm U / 2$ функция $\lambda(\omega)$ меняла знак. Результаты расчета с модельной плотностью состояний (3.26) показаны на рис. 1. Мы видим трехпиковую структуру плотности состояний $\rho(\omega)$ с узким квазичастичным пиком на уровне Ферми. Заметим, что $\operatorname{Im} \Sigma(\omega)$ всюду отрицательна, как и должно быть. При увеличении $U$ интенсивность квазичастичного пика быстро падает до нулевого значения, при этом открывается щель на уровне Ферми (рис. 2). 


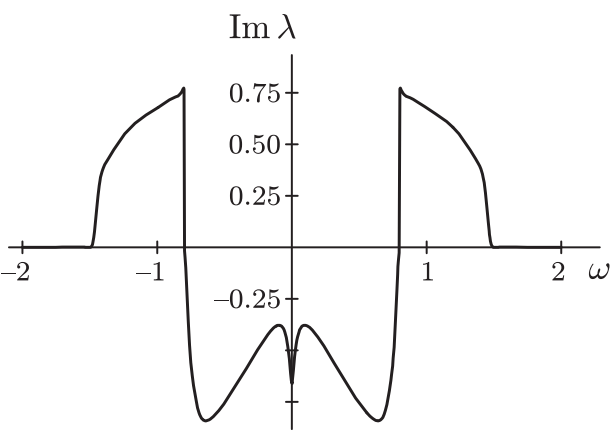

a

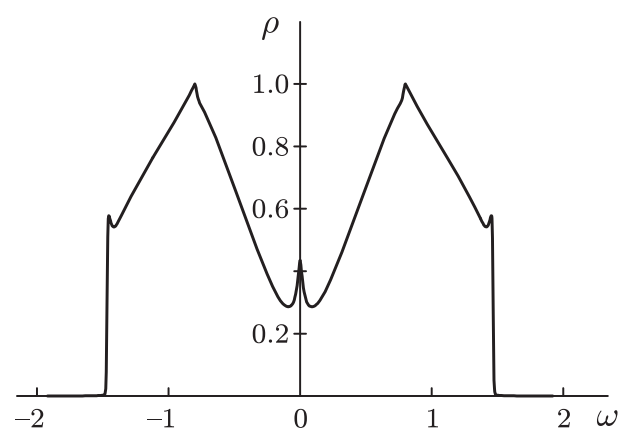

B

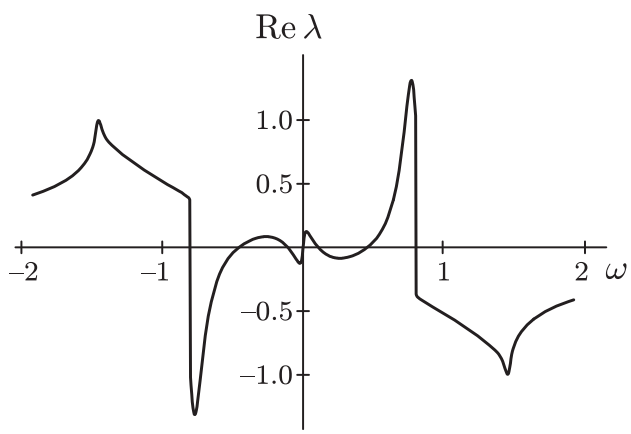

б

$\operatorname{Im} \Sigma$

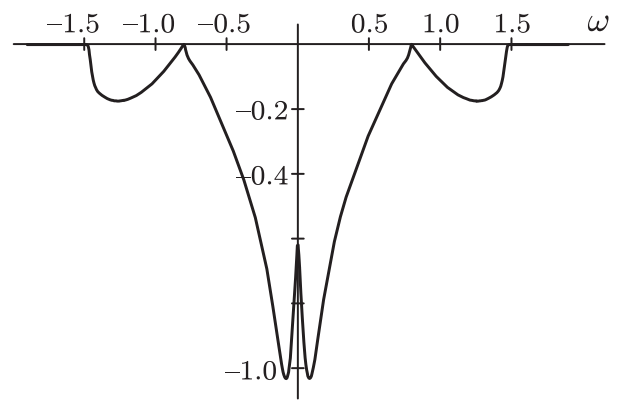

$\Gamma$

Рис. 1. Величины $\operatorname{Im} \lambda(\omega)($ a), $\operatorname{Re} \lambda(\omega)(б), \rho(\omega)($ в) и $\operatorname{Im} \Sigma(\omega)(г)$, рассчитанные для значений параметров $U=1.6, \Gamma=0.01, T=0.001$. Все энергии выражены в единицах гиперзатравочной зоны $W$. Использована прямоугольная плотность состояний в затравочной полосе.

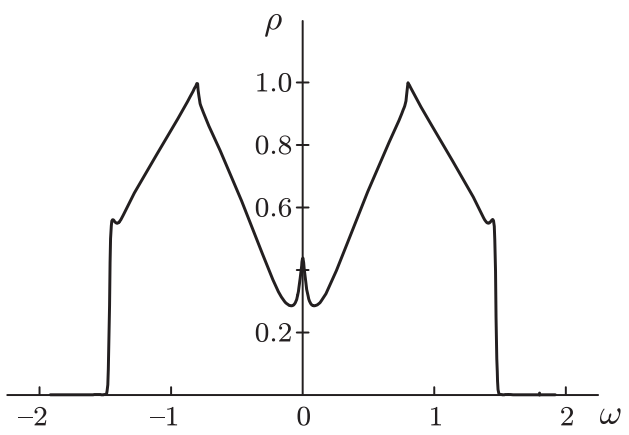

a

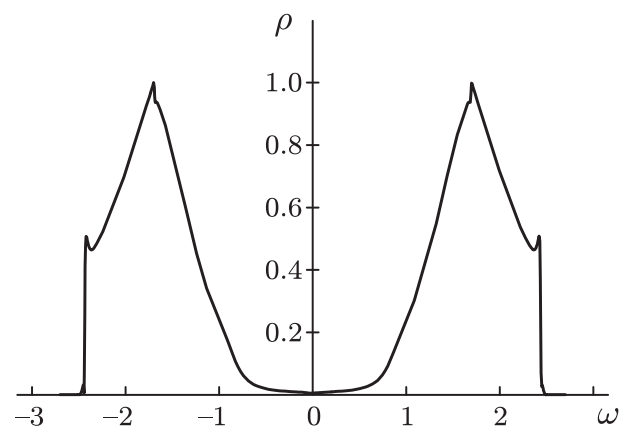

б

Рис. 2. Плотность электронных состояний для значений $U=1.7$ (а) и $U=3.4$ (б). Остальные параметры те же, что и на рис. 1. 


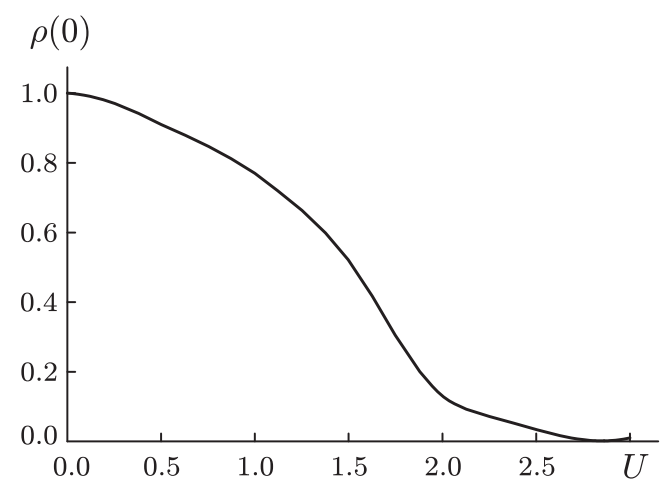

Рис. 3. Плотность электронных состояний на уровне Ферми в зависимости от $U$ при значениях $\Gamma=0.01, T=0.001$.

Как видно из рис. 1б, $\operatorname{Re} \lambda(\omega)$ в окрестности уровня Ферми линейна, причем наклон ее уменьшается с ростом $U$ так, что при критическом значении $U$, когда центральный пик исчезает, угол наклона меняет знак. Острые пики на кривых $\rho(\omega)$ являются следствием прямоугольной формы затравочной плотности состояний. Расчет с полуэллиптической плотностью состояний дает гладкие кривые, но очень похожие на те, которые получены с помощью прямоугольной плотности. Таким образом, модельная плотность затравочных состояний (3.26) качественно не искажает структуру спектра, но позволяет продвинуться дальше в аналитических расчетах (интегрировать по $\varepsilon$ ).

На рис. 3 показано, как уменьшается плотность состояний $\rho(0)$ на уровне Ферми в зависимости от $U$. Переход из металлической в диэлектрическую фазу происходит при $U / W$, слегка превышающем 2. Вычисления показывают, что с ростом температуры интенсивность центрального пика падает. Все отмеченные свойства спектра согласуются с результатами ТДСП, представленными в работах [10], [11] и других, где исследуется модель Хаббарда при половинном заполнении.

Обсудим теперь роль вкладов прямого электронно-электронного рассеяния, описываемых величинами $\varphi^{ \pm}(\omega)$.

На рис. 4 для примера представлены рассчитанные по формулам (3.12) и (3.13) функции $\operatorname{Im} \varphi^{-}(\omega)$ и $\operatorname{Im} m^{-}(\Omega)$. Мы видим, что $\operatorname{Im} m^{-}(\Omega)$ имеет порядок $10^{-2}$, а $\operatorname{Im} \varphi^{-}(\omega)-$ порядок $10^{-3}$, тогда как порядок $\lambda(\omega)$ равен 1 . Величины $\operatorname{Im} \varphi^{+}(\omega)$ и $\operatorname{Im} m^{+}(\Omega)$ с другой симметрией имеют те же порядки, что и $\operatorname{Im} \varphi^{-}(\omega)$ и $\operatorname{Im} m^{-}(\Omega)$, соответственно. При $\omega=0$ все эти величины равны нулю, так что основной вес спектрального распределения для каждой из них приходится на высокие частоты $\omega$ порядка 1 . В силу численной малости величин $\varphi^{ \pm}(\omega)$ ими можно пренебречь везде в уравнениях для электронной $Ф Г$, так что поведение системы определяется исключительно величиной $\lambda(\omega)$, описывающей взаимодействие квазичастиц со спиновыми коллективными модами. 


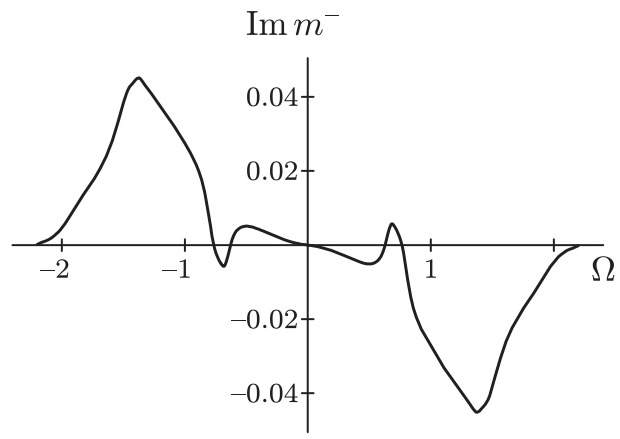

a

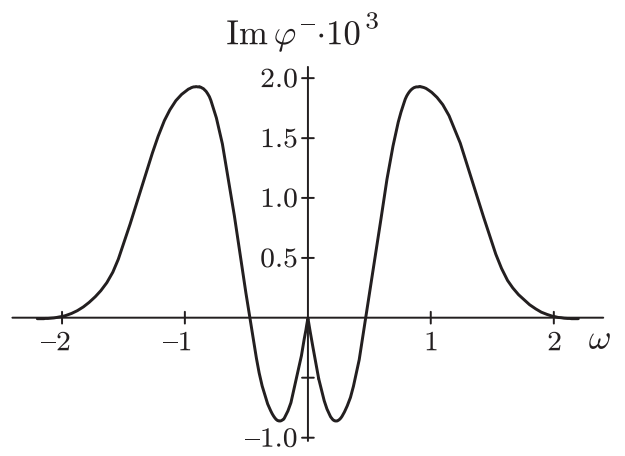

б

Рис. 4. Величины $\operatorname{Im} m^{-}(\Omega)$ (а) и $\operatorname{Im} \varphi^{-}(\omega)$ (б) для параметров $U=1.6$, $\Gamma=0.01, T=0.001$.

\section{5. ЗАКЛЮЧЕНИЕ}

Основной вывод, который можно сделать на основе проведенного исследования, состоит в том, что физика модели Хаббарда при половинном заполнении может быть получена в рамках теории возмущений вблизи атомного предела с собственно-энергетической частью, описывающей взаимодействие электронов со спиновыми коллективными модами. Вклад от процессов рассеяния квазичастиц друг на друге оказывается мал, а вклад, учитывающий взаимодействие с коллективными спиновыми флуктуациями имеет форму вклада в ССБП. Если в интегральных уравнениях для собственно-энергетической части игнорировать импульсную зависимость в смысле предела $d \rightarrow \infty$, решение упрощенных интегральных уравнений воспроизводит основные результаты ТДСП. В частности, при увеличении кулоновского параметра $U$ плотность состояний в электронном спектре эволюционирует от трехпиковой структуры к двухпиковой, обеспечивая фазовый переход металл-изолятор при $U \sim W$; при этом интенсивность центрального квазичастичного пика монотонно падает с ростом $U$. Поведение спектральной плотности с температурой и основными параметрами системы согласуются с основными расчетами ТДСП; основное отличие состоит в меньшей высоте квазичастичного пика.

Однако предлагаемый нами подход дает несколько другой взгляд на проблему. В ТДСП динамика электрона на решетке сводится к некоторой вспомогательной однопримесной задаче. Особенности квазичастичного спектра модели Хаббарда проявляются через спектральную плотность однопримесной модели Андерсона, и трехпиковая или двухпиковая структура решеточной модели соответствуют разным режимам вспомогательной примесной проблемы. При нашем подходе, в сущности, близком ССБП, появление центрального квазичастичного пика обусловлено рассеянием электронов на спиновых флуктуациях, в котором проявляются черты эффекта Кондо.

Заметим, что мы не решали полностью самосогласованную задачу и не вычисляли ФГ спиновых флуктуаций. Вместо этого мы использовали модельную спиновую 
плотность состояний с одним свободным параметром Г, который задавался произвольно. Конечно, ширина спектрального распределения флуктуаций зависит от $U$, и эта зависимость может быть получена только в самосогласованной теории. В ТДСП нет проблемы вычисления отдельно спиновой $Ф Г$, и теория содержит только один параметр $U$. В этом состоит несомненное преимущество ТДСП, за которое приходится расплачиваться необходимостью численного решения однопримесной задачи.

Предложенная схема может быть использована за пределами половинного заполнения, следует помнить только, что в этом случае большую роль должны играть $\eta_{1}$ и $\eta_{2}$ - величины первого порядка по $t$, которые равны нулю при $n=1$. Эти члены играют такую же роль в формировании квазичастичного спектра, что и поправки к среднему полю, учитывающие статические флуктуации заряда и спина, в подходе, предлагаемом в работе [4].

Благодарности. Данная работа поддержана Программой поддержки ведущих научных школ (грант № НШ-4640.2006.2).

\section{Список литературы}

[1] G. Kotliar, S. V. Savrasov, K. Haule, V.S. Oudovenko, O. Parcoletto, C. A. Marianetti, Rev. Mod. Phys., 78:3 (2006), 865.

[2] N. M. Plakida, V.S. Oudovenko, Phys. Rev. B, 59:18 (1999), 11949.

[3] Н. М. Плакида, Л. Антон, С. Адам, Г. Адам, ЖЭЭТФ, 124 (2003), 367.

[4] Н. М. Плакида, В. С. Удовенко, ЖЭТФ, 104 (2007), 230.

[5] Ю. А. Изюмов, Н. И. Чащин, $Ф М M, 97: 3$ (2004), 5.

[6] Yu. A. Izyumov, N. I. Chaschin, D. S. Alexeev, F. Mancini, Eur. Phys. J. B, 45:1 (2005), 69.

[7] Ю. А. Изюмов, Н. И. Чащин, Д. С. Алексеев, Теория сильно коррелированных систем. Метод производящего функционала, РХД, Москва-Ижевск, 2006.

[8] Д. Н. Зубарев, УФН, 71 (1960), 71.

[9] J. Jaklic, P. Prelovsek, Phys. Rev. Lett., 75 (1995), 1340.

[10] A. Georges, G. Kotliar, Phys. Rev. B, 45 (1992), 6479.

[11] R. Bulla, T. A. Costi, D. Vollhardt, Phys. Rev. B, 64 (2001), 045103.

Поступила в редакцию 5.06.2007 